\title{
REM Sleep Behavior Disorder among Veterans with and without Post-Traumatic Stress Disorder
}

\author{
EunYoung Lee ${ }^{1}$, Kiwon Kim¹, Hyung Seok So ${ }^{1}$, Jin Hee Choi ${ }^{1}$, In-Young Yoon ${ }^{2,3}$, and Hayun Choi ${ }^{1 凶}$ \\ ${ }^{1}$ Department of Psychiatry, Veteran Health Service Medical Center, Seoul, Republic of Korea \\ ${ }^{2}$ Department of Neuropsychiatry, Seoul National University Bundang Hospital, Seongnam, Republic of Korea \\ ${ }^{3}$ Department of Psychiatry, Seoul National University College of Medicine, Seoul, Republic of Korea
}

\begin{abstract}
Objective Among veterans, the prevalence of rapid eye movement sleep behavior disorder (RBD) is higher than among the general population, and some evidence suggests that this is related to post-traumatic stress disorder (PTSD). The purpose of this study was to determine whether the frequency of RBD differs depending on the presence of PTSD or trauma.

Methods Patients who underwent nocturnal polysomnography (PSG) and sleep-related questionnaire surveys at the Veteran Health Service Medical Center were reviewed retrospectively. Based on patients with PTSD ( $n=20 ; 100 \%$ male; $67.9 \pm 8.5$ years of age), we matched patients exposed to trauma without PTSD (n23; 100\% male; age 64.0 \pm 13.4$)$ and patients without trauma ( $\mathrm{n}=21 ; 100 \%$ male; age $59.86 \pm 10.9)$.

Results PTSD patients reported dream enactment behavior more than the trauma-exposed group without PTSD or the control group $(\mathrm{p}=0.006)$. After adjusting for age, there were more RBD patients in the PTSD group than in the trauma exposed group ( $\mathrm{p}=0.049$ ).

Conclusion The results showed that RBD occurred significantly more in veterans with PTSD than those exposed to trauma, which suggests that there may be a pathophysiological association between PTSD and RBD.

Psychiatry Investig 2020;17(10):987-995
\end{abstract}

Key Words PTSD, Veterans, REM sleep behavior disorder.

\section{INTRODUCTION}

Post-traumatic stress disorder (PTSD) is a mental disorder that can occur after an individual has experienced or witnessed death, a near death experience, serious injuries, or sexual violence. ${ }^{1,2}$ The lifetime prevalence of PTSD varies from $1.9 \%$ to $8.8 \%$ as per various studies, ${ }^{3,4}$ but is more commonly found among war veterans. ${ }^{2}$ The main clinical features of PTSD are generally grouped into four categories according to the DSM5: recurrent intrusion symptoms of traumatic events, avoidance of trauma-related stimuli, negative alterations in cognition and mood related to trauma, and marked alterations in arousal and reactivity associated with the traumatic events. ${ }^{1}$ Most patients with PTSD complain of sleep problems, includ-

Received: June 5, 2020 Revised: August 4, 2020

Accepted: August 20, 2020

$\triangle$ Correspondence: Hayun Choi, MD

Department of Psychiatry, Veteran Health Service Medical Center, 53 Jinhwangdo-ro 61-gil, Gangdong-gu, Seoul 05368, Republic of Korea

Tel: +82-2-2225-1158, Fax: +82-2-2225-3947, E-mail: chhy0402@gmail.com

(a) This is an Open Access article distributed under the terms of the Creative Commons Attribution Non-Commercial License (https://creativecommons.org/licenses/bync/4.0) which permits unrestricted non-commercial use, distribution, and reproduction in any medium, provided the original work is properly cited. ing trauma-related distressing dream content and difficulty in achieving or maintaining sleep. ${ }^{5-9}$ The presence of sleep problems increases the severity of PTSD symptoms and the severity of the accompanying moods and other symptoms..$^{10-12}$

Sleep disorders have also been reported among veterans without PTSD; the most common being insomnia and trauma-related nightmares (TRN). ${ }^{13}$ According to Raskind et al. ${ }^{14}$ TRN are predominantly expressed during light sleep, interfere with rapid eye movement (REM) sleep, and are often accompanied by destructive nocturnal behavior, including dream enactment behavior or activation of the autonomic sympathetic nerve. ${ }^{14}$ Various movements are seen in the TRN range, from overtly aggressive behavior to REM sleep without atonia (RSWA) during polysomnography (PSG) ${ }^{15,16}$ In practice, it is highly likely that RBD and TRN are not distinguishable..$^{16,17}$ Dream enactment behavior (DEB) on medical history and RSWA on PSG are included in the diagnostic criteria for both RBD and TRN. ${ }^{18}$ In addition, the prevalence of idiopathic RBD among the general population over 60 years of age was $~ 2 \%$ to $\sim 5.5 \%,{ }^{19,20}$ but it was found to be $56 \%$ among a small-population study of PTSD patients. ${ }^{21}$ In this regard, some researchers suggest that PTSD and RBD are not two distinct clinical 
entities, but rather that their interaction can lead to the expression of preexisting synuclein pathology. ${ }^{17}$

Therefore, the purpose of this study was to identify the characteristics and differences of sleep patterns in each group by comparing the results of the nocturnal polysomnography and sleep-related questionnaires of the three groups. The groups included: a group of patients with PTSD; a group of patients with trauma exposure but not yet diagnosed with PTSD, and a group without a history of trauma exposure (control group). The hypothesis of this study is as follows. First, that RBD will be more common among the PTSD group than the other two groups, and this will be related to the presence or absence of PTSD. Second, RBD will be more common among the trauma exposed group without PTSD than in the control group, and this will be associated with the presence or absence of traumatic exposure.

\section{METHODS}

\section{Participants}

Between July 2012 to May 2019, 2,262 patients visited the Psychiatry department of the Veterans Health Service Medical Center and underwent an in-lab video assisted nocturnal PSG. From among those, we selected patients with PTSD, trauma exposed patients without PTSD, and patients without trauma (control group) and analyzed this sample. Psychiatrists diagnosed PTSD during a clinical interview. Among the study's participants, those with schizophrenia, delusional disorder, delirium, dementia or a mental disorder due to medical conditions were excluded from the study. Those with a history of neurological abnormalities, such as head trauma and convulsive disease were also excluded.

We selected 20 patients with PTSD who fulfilled the inclusion criteria and tried to match trauma exposed patients without PTSD, and patients without trauma based on age and sex with the PTSD group by random sampling (Figure 1). Since the PTSD patients were all males, all subjects were males as a result of sex matching. The age distribution in the control group could not be matched with that in the PTSD group, and satisfactory results were obtained only at the significance level of 0.1 . The reason why it was difficult to match the age distribution between the three groups was that patients in the control group were mostly descendants or spouses of veterans. Since most of the spouses of veterans were female, they were not matched as control group. Since many of the patients without trauma were descendants of veterans, they had to be younger than the veterans. This study is a retrospective study that was conducted through the review of medical records and was approved by the Institutional Review Board of the Veterans Health Service Medical Center (IRB No. 2019-06-002). Since it was a retrospective study, informed consent from participants was waived off by the Institutional Review Board of the Veterans Health Service Medical Center.

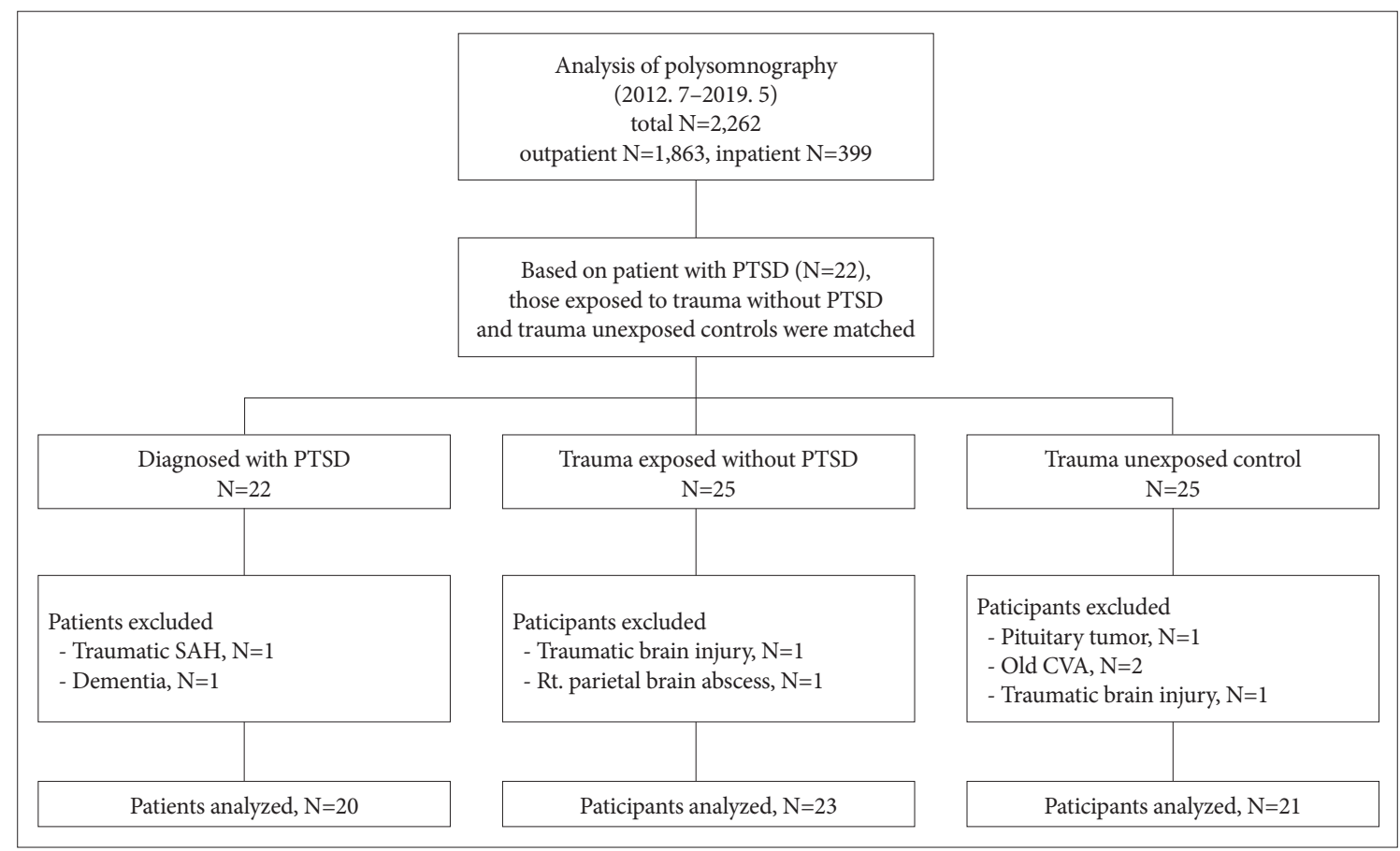

Figure 1. Schematic overview of our patient population. PTSD: posttraumatic stress disorder, SAH: subarachnoid hemorrhage, CVA: cerebrovascular accident. 


\section{Clinical information and neuropsychological evaluation}

All participants had an outpatient interview with a Psychiatrist prior to the PSG and completed a self-report questionnaire. The contents of the questionnaire included personal and clinical information such as age, gender, education, weight, height, body mass index (BMI), sleep-related lifestyle, past medical history, current medication, and sleeping habits. A clinical psychological evaluation was made using the Pittsburgh Sleep Quality Index, ${ }^{22}$ the Epworth Sleepiness Scale, ${ }^{23}$ the Stanford Sleepiness Scale, ${ }^{24}$ and the Insomnia Severity Index, ${ }^{25}$ as well as a Korean version-a Dysfunctional Beliefs and Attitudes about Sleep Questionnaire-16, ${ }^{26}$ and Beck's Depression Inventory- $\mathrm{II}^{27}$ to evaluate daytime sleepiness, subjective sleep quality, and depression during the day.

Participants completed the Pittsburgh Sleep Quality Index (PSQI) to evaluate subjective sleep quality. PSQI can assess for subjective sleep quality, sleep latency, sleep duration, sleep efficiency, sleep disturbance, sleeping medication use, and daytime dysfunction. ${ }^{22,28,29}$ PSQI consists of 19 questions, and a total index over 5 was regarded as a subjective sleep complaint. ${ }^{22,28,29}$ The Epworth Sleepiness Scale (ESS) is a questionnaire that evaluates the symptoms of daytime sleepiness. It consists of 8 items, and clinically meaningful daytime sleepiness is indicated by 11 points and over. ${ }^{23}$ The Stanford Sleepiness Scale (SSS) is also a patient-reported questionnaire that evaluates the degree of daytime sleepiness. It is rated on a scale of 1 to 7 points, and in cases over 3, sleep debt exists and more sleep is considered necessary. ${ }^{24,26}$ The Insomnia Severity Index (ISI), which is a test for evaluating the severity of insomnia, has a total of 7 questions and is a 28-point test. A score of $0-7$ points indicates almost no insomnia, 8-14 points indicates subthreshold insomnia, 15-21 points indicates moderate insomnia, and 22-28 points indicates severe insomnia; a score of 15 or more can be evaluated as clinically meaningful insomnia. ${ }^{25}$ The Korean version-Dysfunctional Beliefs and Attitudes about Sleep Questionnaire (K-DBAS-16) is a patient-reported test for evaluating negative perception of sleep which consists of 16 questions and has a scale of up to 10 points (highly agreed) at a minimum of zero (highly opposed) for each question. To achieve a reversal of negative perception of sleep could be the goal of cognitive behavioral therapy for insomnia. ${ }^{26}$ In addition, the Beck's Depression Inventory-II (BDI-II) is a patientreported test that screens for depression and consists of 21 questions, which can be rated from 0 to 3 points per question, with a maximum of 63 points and a minimum of 0 , suggesting a state of mild depression at $14-19$ points, ${ }^{27}$ a state of moderate depression at 20-28 points, and a state of severe depression at 29-63 points.

\section{Nocturnal polysomnography}

The polysomnography device used in this study was Comet-PLUS PSG (Grass Instrument Co., Warwick, RI, USA), and standardized electrodes and detectors were used. According to the international 10-20 electrode placement system, electrodes were applied at F4/A1, F3/A2, C4/A1, C3/A2, O1/A2 and $\mathrm{O} 2 / \mathrm{A} 1$, and two electrooculography electrodes were applied on the sides of both eyes to record horizontal and vertical eye movement. The electromyography electrodes were applied at the submentalis muscle and at both anterior tibialis muscles to record the movement of the lower extremities during sleep. Strain gauges were used to detect thoracic and abdominal respiratory movements, and nasal airflow was measured using a nasal pressure cannula. The level of oxygen saturation in the blood was measured at the tip of the second finger of the left hand (index finger) by attaching a pulse oximeter. The sleep structure was analyzed based on standardized reading criteria published by the American Academy of Sleep Medicine. Measurements of nocturnal polysomnography include total sleep time, wake after sleep onset, sleep latency, sleep efficiency, stage N1 sleep time, stage N2 sleep time, stage N3 sleep time, REM sleep time, oxygen desaturation index (ODI), apnea-hypopnea index (AHI), RSWA, and the periodic limb movement index.

\section{Statistical analysis}

SPSS Statistics version 16.0 (SPSS Inc., Chicago, IL, USA) was used to analyze the demographic characteristics and subjective scales of all participants and the values of various PSG variables. To verify normality with the Kolmogorov-Smirnov test, one-way ANOVA, and Kruskal-Wallis tests were used to compare continuous variables between three groups, and chisquared test was used for categorical variables. To evaluate factors related to RSWA, multiple logistic regression using a step selection method was performed. The statistical significance level was defined as when the bidirectional p-value was less than 0.05 .

We defined 'RBD' patients as people who reported DEB in their medical history and in whom RSWA was observed on PSG. We identified several confounding factors using ANOVA, chi-square test results and previous studies. These confounding factors were age, BDI II score, AHI, ODI, neurological and psychiatric comorbid diseases, antidepressants, and use of benzodiazepines and antipsychotics. ${ }^{18,30}$ Of the aforementioned factors, confounders such as age, BDI II score, AHI and ODI were continuous variables, while neurological and psychiatric comorbid diseases, and use of antidepressants, benzodiazepines, and antipsychotics were categorical variables. We performed a multiple regression analysis by setting variables with a p-value of less than 0.05 as predictors in the 
result of simple regression analysis. We used stepwise selection methods in a multiple regression analysis. In the categorical variable coding process, the trauma exposed group without PTSD was designated as a reference.

\section{RESULTS}

\section{Demographic and clinical characteristic findings}

A total of 64 patients participated in the study, 20 of whom were diagnosed with PTSD, 23 were exposed to trauma but not diagnosed with PTSD, and 21 who were not exposed to trauma. Demographic comparison between the three groups did not show significant differences except in BMI, duration of education, psychiatric comorbid diseases, and age. The mean age was 67.85 \pm 8.54 years in the PTSD group and $63.96 \pm 13.35$ years in the exposed group without PTSD, while it was $59.86 \pm 10.90$ years in the unexposed group (control), showing a difference between the three groups $(\mathrm{p}=0.036)$. Evaluation of clinical symptoms on the self-reported questionnaires showed that there were differences between the three groups on the Epworth Sleepiness Scale (ESS), the Stanford Sleepiness Scale (SSS), and the Insomnia Severity Index (ISI), a Korean versiona Dysfunctional Beliefs and Attitudes about Sleep Questionnaire-16 (K-DBAS-16) and Beck's Depression Inventory-II (BDIII), the use of psychiatric drugs, and the reason for PSG. DEB occurred among 11 people in the PTSD group (55.0\%), which was more than in the other two groups $(\mathrm{p}=0.006)$ (Table 1$)$.

\section{Sleep-related variables during nocturnal polysomnography}

During nocturnal PSG, there were no significant differences between the three groups in sleep patterns, including total

Table 1. Demographic and clinical characteristic findings of eligible participants

\begin{tabular}{|c|c|c|c|c|}
\hline & PTSD $(\mathrm{N}=20)$ & Trauma-exposed $(\mathrm{N}=23)$ & Control $(\mathrm{N}=21)$ & $\mathrm{p}$ \\
\hline Age, yr & $67.85(8.54)^{*}$ & $63.96(13.35)$ & $59.86(10.90)$ & 0.036 \\
\hline $\mathrm{BMI}, \mathrm{kg} / \mathrm{m}^{2}$ & $24.66(2.98)$ & $25.89(3.73)$ & $25.71(3.33)$ & 0.454 \\
\hline Education & $11.74(3.00)$ & $13.27(2.33)$ & $12.40(3.63)$ & 0.29 \\
\hline PSQI & $9.37(3.77)$ & $9.78(4.10)$ & $7.11(4.12)$ & 0.085 \\
\hline SSS & $3.42(1.46)^{*}$ & $3.05(1.17)$ & $2.25(1.16)$ & 0.005 \\
\hline ESS & $7.63(5.08)$ & $10.48(5.43)^{*}$ & $6.67(4.90)$ & 0.045 \\
\hline ISI & $13.26(6.68)^{*}$ & $11.39(6.65)$ & $6.40(7.32)$ & 0.003 \\
\hline DBAS & $6.39(1.85)^{*}$ & $5.59(1.74)$ & $4.52(1.74)$ & 0.007 \\
\hline BDI & $19.68(11.91)^{*}$ & $12.83(8.61)^{*}$ & $7.05(6.13)$ & 0.001 \\
\hline \multicolumn{5}{|l|}{ Cormobidity } \\
\hline Cardiovascular ds & $11(57.9)$ & $18(78.3)$ & $11(55.0)$ & 0.217 \\
\hline Neurologic ds & $4(21.1)$ & $2(8.7)$ & $1(5.0)$ & 0.315 \\
\hline Pulmonary ds & $3(15.8)$ & $2(8.7)$ & $1(5.0)$ & 0.575 \\
\hline Gastrointestinal ds & $7(36.8)$ & $10(43.5)$ & $4(20.0)$ & 0.254 \\
\hline Endocrine ds & $8(42.1)$ & $13(56.5)$ & $4(20.0)$ & 0.051 \\
\hline Urological ds & $13(68.4)$ & $14(60.9)$ & $9(45.5)$ & 0.315 \\
\hline Other psychiatric ds & $15(78.9)$ & $4(17.4)$ & $1(5.0)$ & $<0.001$ \\
\hline \multicolumn{5}{|l|}{ Medication } \\
\hline Antidepressants & $16(80.0)$ & $4(17.4)$ & $2(9.5)$ & $<0.001$ \\
\hline Benzodiazepines & $14(70.0)$ & $4(17.4)$ & $3(14.3)$ & $<0.001$ \\
\hline Antipsychotics & $9(45.0)$ & $0(0.0)$ & $0(0.0)$ & $<0.001$ \\
\hline Reason for PSG & & & & 0.006 \\
\hline Dream enactment behavior & $11(55.0)$ & $4(17.4)$ & $5(23.8)$ & \\
\hline Snoring/apnea & $6(30.0)$ & $19(82.6)$ & $15(71.4)$ & \\
\hline Others & $3(15.0)$ & $0(0.0)$ & $1(6.3)$ & \\
\hline
\end{tabular}

Data are presented as mean (SD) or N (\%). *vs control group, $\mathrm{p}<0.05$. PTSD: posttraumatic stress disorder, BMI: Body Mass Index, PSQI: Pittsburgh Sleep Quality Index, SSS: Stanford Sleepiness Scale, ESS: Epworth Sleepiness Scale, ISI: Insomnia Severity Index, DBAS: Korean versionDysfunctional Beliefs and Attitudes about Sleep Questionnaire-16, K-DBAS-16, BDI: Beck Depression Inventory-II, ds: disease, PSG: polysomnography 
sleep time, sleep latency, sleep efficiency, sleep stage fraction, apnea-hypopnea index, and periodic limb movement with arousal. There were differences between the three groups on RSWA ( $\mathrm{p}=0.026)$ and ODI ( $\mathrm{p}=0.019)$. RSWA appeared more among the PTSD group than in the trauma exposed group without PTSD. However, contrary to our hypothesis, there was no difference between the trauma and the control group. The average ODI was higher among the control group than the PTSD group (Table 2).

\section{Binary logistic regression}

Logistic regression analysis was performed to analyze the effects of PTSD or trauma exposure on abnormal sleep, including RBD. There were eight RBD patients in the PTSD group, one in the trauma exposed group without PTSD, and four in the control group. Groups, age, BDI II score, AHI, ODI, neurological and psychiatric comorbid diseases, antidepressants, benzodiazepines, and antipsychotics entered in the first place of the stepwise selection methods, and groups, age and AHI were remained at the last step. Further, if age and AHI were controlled, the PTSD group was found a stronger predictor of RBD compared to the trauma exposed group without PTSD $(\mathrm{p}=0.049)$ (Table 3). When we set the predictors as PTSD and trauma exposure factors instead of groups and performed multiple regression analysis, the PTSD factor showed a cor- relation with RBD after considering age and AHI $(\mathrm{p}=0.049)$.

\section{DISCUSSION}

This study investigated sleep patterns using nocturnal PSG and sleep-related questionnaires among veterans with and without PTSD. The average age of the PTSD group was 8 years older than that of the non-exposed group. As shown by the self-report questionnaire, SSS, ISI, K-DBAS-16, and BDI II scores were significantly higher among the PTSD group than the other two groups. This suggests that people with PTSD were obviously aware of having insomnia and daytime interference caused by it, often had irrational beliefs toward sleep, and depression. ${ }^{31-33}$ The results of PSG, ODI and RSWA showed significant differences between the three groups. RSWA was significantly more frequent among those with PTSD than the control group, and ODI was significantly lower among the control group or the trauma-exposed group without PTSD compared to the PTSD group.

Of the 43 traumatic exposure participants included in the study, 29 (67.4\%) had suffered physical and mental trauma associated with the Vietnam War, while 12 (27.9\%) had military-related trauma due to injuries or explosions during military training. Two out of the 12 were special mission performers, ${ }^{34}$ and two (4.7\%) of the total trauma exposure participants

Table 2. Polysomnographic findings among eligible participants

\begin{tabular}{|c|c|c|c|c|}
\hline & PTSD $(\mathrm{N}=20)$ & Trauma-exposed $(\mathrm{N}=23)$ & Control $(\mathrm{N}=21)$ & $\mathrm{p}$ \\
\hline TST, min & $298.11(60.67)$ & $304.94(72.07)$ & $315.28(52.93)$ & 0.685 \\
\hline WASO, min & $91.91(55.64)$ & $103.73(77.86)$ & $74.46(52.54)$ & 0.318 \\
\hline Sleep latency, min & $39.15(52.13)$ & $21.72(35.36)$ & $16.31(17.35)$ & 0.349 \\
\hline Sleep efficiency, \% & $70.42(16.40)$ & $71.32(17.74)$ & $77.98(13.61)$ & 0.323 \\
\hline Stage $1, \%$ & $15.86(8.64)$ & $16.29(9.95)$ & $15.77(9.96)$ & 0.972 \\
\hline Stage $2, \%$ & $58.91(16.71)$ & $53.54(13.27)$ & $59.43(10.23)$ & 0.288 \\
\hline Stage $3, \%$ & $4.75(9.47)$ & $9.58(12.88)$ & $6.67(6.79)$ & 0.159 \\
\hline REM, \% & $20.48(10.82)$ & $20.57(9.69)$ & $18.14(8.20)$ & 0.648 \\
\hline AHI & $22.45(22.71)$ & $32.71(22.17)$ & $36.32(25.43)$ & 0.067 \\
\hline NRAHII & $22.21(23.63)$ & $34.72(25.94)$ & $36.41(26.56)$ & 0.102 \\
\hline RAHII & $22.34(18.57)$ & $24.67(13.78)$ & $37.87(28.04)$ & 0.158 \\
\hline ODI, events/hr & $8.97(10.93)^{*}$ & $15.38(10.21)$ & $20.75(16.71)$ & 0.019 \\
\hline $\mathrm{SaO} 2<90, \%$ & $4.95(9.35)$ & $5.24(6.44)$ & $9.33(14.42)$ & 0.154 \\
\hline $\mathrm{MinSaO} 2, \%$ & $83.75(6.89)$ & $82.48(5.83)$ & $79.71(7.55)$ & 0.154 \\
\hline PLM Ar & $4.43(11.44)$ & $1.29(3.99)$ & $1.89(3.59)$ & 0.373 \\
\hline PLMI & $18.25(26.09)$ & $8.20(17.91)$ & $16.92(30.78)$ & 0.441 \\
\hline RSWA & $9(45.0)^{\dagger}$ & $2(8.7)$ & $6(28.6)$ & 0.026 \\
\hline
\end{tabular}

Data are presented as mean (SD) or $\mathrm{N}(\%){ }^{*}$ vs control group, $\mathrm{p}<0.05,{ }^{\dagger}$ vs trauma group, $\mathrm{p}<0.05$. TST: total sleep time, WASO: wake after sleep onset, REM: rapid eye movement, AHI: apnea-hypopnea index, NRAHI: non rapie eye movement apnea-hypopnea index, RAHI: rapid eye movement apnea-hypopnea index, ODI: oxygen desaturation index, $\mathrm{SaO} 2$ : oxygen saturation, PLM Ar: periodic limb movements with arousal, PLMI: periodic limb movement index, RSWA: rapid eye movement sleep without atonia 
Table 3. Binary logistic regression analysis

\begin{tabular}{|c|c|c|c|c|c|}
\hline \multirow{2}{*}{ Outcome } & \multirow{2}{*}{ Predictor } & \multicolumn{2}{|l|}{ Simple } & \multicolumn{2}{|l|}{ Multiple } \\
\hline & & OR $(95 \% \mathrm{Cl})$ & $\mathrm{p}$ & OR $(95 \% \mathrm{Cl})$ & $\mathrm{p}$ \\
\hline \multirow[t]{11}{*}{ RBD } & PTSD group* & $14.667(1.634-131.657)$ & 0.016 & $11.101(1.004-122.771)$ & 0.049 \\
\hline & Control group* & $5.176(0.529-50.653)$ & 0.158 & $12.921(0.965-172.953)$ & 0.053 \\
\hline & Age & $1.186(1.041-1.352)$ & 0.010 & $1.234(1.041-1.464)$ & 0.015 \\
\hline & AHI & $0.935(0.886-0.935)$ & 0.010 & $0.960(0.916-1.006)$ & 0.089 \\
\hline & ODI & $0.901(0.827-0.981)$ & 0.016 & & \\
\hline & BDI & $1.014(0.957-1.074)$ & 0.639 & & \\
\hline & Neurologic ds & $3.375(0.651-17.509)$ & 0.148 & & \\
\hline & Other psychiatric ds & $3.231(0.915-11.406)$ & 0.068 & & \\
\hline & Antidepressants & $0.167(0.806-9.731)$ & 0.105 & & \\
\hline & Benzodiazepines & $3.083(0.882-10.782)$ & 0.078 & & \\
\hline & Antipsychotics & $2.200(0.0469-10.331)$ & 0.318 & & \\
\hline
\end{tabular}

Data are presented as mean (CI); ${ }^{*} 95 \%$ CI does not overlap null value of $1 .{ }^{*}$ using Trauma exposed group without PTSD as reference. PTSD: posttraumatic stress disorder, BDI: Beck Depression Inventory-II, AHI: apnea hypopnea index, ODI: oxygen desaturation index

had trauma related to a traffic accident. By referring to their medical records, we discovered that these traumatic events occurred in their early twenties, making it possible to specify the timing of the trauma exposure. The time elapsed between trauma exposure and PSG was, on average, approximately 41 $[41.05, \mathrm{SD}(1.71)]$ years, and there was no significant difference in the length of this period between the veterans with or without PTSD. Participants with PTSD were rarely diagnosed immediately after the trauma. PTSD symptoms such as hypervigilance, intrusive symptoms, and sleep disorders started or worsened more than 40 years after trauma exposure. Various factors affecting PTSD can be divided into pre-traumatic factors (former exposure to trauma, low socio-economic status and education level, age at the time of trauma, etc.), factors during trauma (intensity of trauma, severity of life threats, etc.), and post-traumatic factors (inappropriate coping skills, negative life events after trauma, social support). ${ }^{34}$ Considering the socio-economic history of the Republic of Korea, factors such as low socio-economic status, insufficient social-psychological support, and difficulty in returning to daily life may have acted as risk factors that made these veterans vulnerable to PTSD. These post-traumatic factors may have significantly contributed to the PTSD of patients in this study, and their symptoms exacerbated after a sudden life change such as retirement. In a longitudinal study on veterans of the U.S.-Vietnam War, the number of late onset PTSD patients since 2012 was reported to be more than twice that of the chronic PTSD patients diagnosed with it in $1992 .{ }^{35}$

A total of 2,262 patients underwent PSG from July 2012 to May 2019 at the psychiatry department of Veteran Health Service Medical Center. Only 23 patients were diagnosed with PTSD among them. Considering that the prevalence rate of
PTSD is about $8 \%^{4}$ (10.50\% amongst theater veterans and $4.45 \%$ amongst nontheater veterans), ${ }^{35}$ the prevalence rate of PTSD in this research was much less at about $1 \%$. The reason behind this is that it was not possible to conduct PSG to evaluate sleep disorders among all patients with PTSD. Patients with extreme sleep difficulties do not want to sleep with multiple sensors attached to their bodies in an unfamiliar environment. In some cases, the patient may feel it is extremely difficult to sustain the PSG and may have to stop in the middle of the test. In addition, PTSD participants usually complained of insomnia and additional abnormal behavioral symptoms during sleep. Therefore, they may not have been actively advised to perform a PSG as part of their diagnosis.

In Table 2, AHI was over 20 among the PTSD group, but relatively few participants in this group complained of symptoms related to sleep apnea. While AHI did not show a difference between groups, ODI was significantly lower among the PTSD group than the non-exposed group. According to a study by Huang et al., ${ }^{36}$ patients with RBD and OSA had no difference in AHI compared to those with OSA alone, but ODI tended to be lower, which suggests the possibility that excessive EMG activity among RBD patients protected them from severe OSA. ${ }^{36}$ In this study, the lower ODI among the PTSD group compared to the non-exposed group was likely due to increased EMG activity during REM sleep.

Excessive sleep discomfort among PTSD patients was not observed with the PSG. The PTSD group perceived their insomnia as being significantly more severe than the control group but no significant difference in the total sleep time or sleep efficiency was observed in the PSG between the three groups. This was because patients with severe PTSD symptoms may have been excluded from this study due to the afore- 
mentioned difficulties in undergoing PSG and the likelihood of them stopping a PSG test midway. Although PTSD patients are known for their tendency to be more vulnerable and hypervigilant, ${ }^{33}$ which can lead to greater night-time alertness, studies into objectively measured sleep indicators among PTSD patients have been inconsistent. ${ }^{37}$ There are studies where PTSD patients overestimated sleep disturbance in sleep diaries or questionnaires compared to PSG findings, while others have underestimated it in studies using actigraphy. Insomnia patients tend to exaggerate their sleep patterns in comparison with the variables observed in PSG (e.g., underestimation of total sleep time and overestimation of sleep latency). ${ }^{33,37-39}$

Although the association between PTSD and RBD was confirmed in this study as with previous studies, whether PTSD plays a causal role in the development of RBD remains uncertain. ${ }^{15-18,21,40}$ In PTSD, most nightmares and awakenings occur during and interfere with REM sleep. ${ }^{41}$ The REM sleep state provides conditions that help to modify the memory network that separates events from emotional excitement in order to aid emotional adaptation, and some argue that this process appears to be impaired among patients with PTSD. ${ }^{42}$ Pathophysiologically, the rate of replacement of norepinephrine in PTSD cases increases, leading to depletion of norepinephrine in the locus coeruleus ${ }^{18}$ and a reduction of nerve activity due to the death of neurons in the locus coeruleus. Studies have indicated that this not only causes PTSD-related symptoms, but also exhibits the suppression of the pedunculopontine nucleus, as seen among patients with RBD, Parkinson's disease, and Lewy body dementia. ${ }^{18,43}$

The presence of RSWA on the PSG showed no difference between the trauma group or the control group. In terms of $\mathrm{RBD}$ as the dependent variable, the trauma group did not show any difference compared to the non-exposed group. Frequent respiratory disturbances among the elderly were likely to affect electromyogram(EMG), although the PSG readings in this study were based on the AASM criteria. ${ }^{36}$ In the trauma exposure group without PTSD, there may be a process which inhibits the neuropathological pathway that can produce RBD. ${ }^{44}$ Furthermore, control group participants may have undiagnosed secondary PTSD, as they are mostly members of the families of veterans with past combat exposure. ${ }^{45}$ In addition, the veterans at our hospital have a self-pay rate of 0 to $10 \%$, whereas the family members of the veterans have a self-pay rate of 40 to $50 \%$. Therefore, if a veteran's family member comes to see a doctor, the perceived severity of the disease may be worse than that of veterans.

This study has several limitations. First, the number of participants in the study was small, which limits the interpretation of the results. We started the study with the expectation that there would be many PTSD patients who underwent PSG, but the number of PTSD patients who met the inclusion criteria was fewer than expected. Although there was a positive correlation between the presence or absence of PTSD and $\mathrm{RBD}$, which were the main outcome of interest of this study, there is a limitation that the $\mathrm{p}$-value shows a marginal significance level of 0.049 . There is a lack of generalization of the sample since most of the participants are elderly veterans in the late 50 s to late 60 s. While the fact that this study was conducted in one hospital suggests there are limitations to its representativeness, there were more patients who have been exposed to combat in this hospital than in other hospitals in South Korea, strengthening its suitability to the research topic. In addition, we tried to match the three groups by age in the first place, but most male patients without trauma were descendants of veterans and the study groups could not be matched. So we added 'age' to the analysis model as a confounding factor. Limitations might arise in determining the direct relevance between a patient's trauma and their sleep disorder due to the fact that more than 40 years had passed since the majority of participants' trauma exposure. However, since RBD tends to be invoked when individuals are in their late 50s and late 60s, these participants were nonetheless suited to research related to the existence of RBD. ${ }^{46}$ As the study included participants who were prescribed antidepressants, benzodiazepines, and antipsychotics, these drugs may have had an effect on the research results. ${ }^{8,15,18,47-49}$ However, the effect of these drugs on RBD was not found to be statistically significant. Furthermore, as most PTSD patients take psychiatric drugs, this is reflective of realistic medical situations. As PSG analysts were not blinded in terms of the patients' clinical history and diagnosis, the patients' history may have affected their analysis of RSWA. Additionally, there were difficulties in clearly distinguishing TRN from RBD because it was difficult to identify the automatic hyperarousal which is observed in TRN. ${ }^{16-18,43}$ Lastly, the severity of trauma was not included in the analysis because there were only 9 subjects who performed Clinicianadministered PTSD Scale (CAPS) and the PTSD Checklist (PCL) in PTSD group.

Despite its limitations, the study provides a meaningful comparison of the sleep patterns of PTSD groups, trauma exposure groups without PTSD, and unexposed groups. The results showed that there were more RBDs among the PTSD group than in the traumatic exposure group, indicating that there may be a pathological link between PTSD and RBD. Further research is needed to establish a relationship between RBD and PTSD.

\section{Acknowledgments}

This study was supported by the VHS Medical Center Research Grant, Republic of Korea (Grant number: VHSMC 19040). 


\section{Conflicts of Interest}

The authors have no potential conflicts of interest to disclose.

\section{Author Contributions}

Conceptualization: Hayun Choi, Kiwon Kim, In-Young Yoon. Data curation: EunYoung Lee. Formal analysis: EunYoung Lee, Hayun Choi. Funding acquisition: Hayun Choi. Investigation: EunYoung Lee. Methodology: EunYoung Lee, Hayun Choi. Project administration: EunYoung Lee, Hayun Choi. Resources: Hayun Choi, Kiwon Kim, Hyung Seok So, Jin Hee Choi. Software: EunYoung Lee, Hayun Choi. Supervision: Hayun Choi, In-Young Yoon. Validation: EunYoung Lee. Visualization: EunYoung Lee, Hayun Choi. Writing_original draft: EunYoung Lee. Writing_review \& editing: Hayun Choi, Kiwon Kim, Hyung Seok So.

\section{ORCID iDs}

EunYoung Lee

Kiwon Kim

Hyung Seok So

Jin Hee Choi

In-Young Yoon

https://orcid.org/0000-0003-2113-1942

https://orcid.org/0000-0001-7594-0142

https://orcid.org/0000-0001-5470-8707

https://orcid.org/0000-0002-8981-3168

Hayun Choi

ps://orcid.org/0000-0002-3995-8238

https://orcid.org/0000-0002-8404-8006

\section{REFERENCES}

1. American Psychiatric Association. Diagnostic and Statistical Manual of Mental Disorders, 5th Edition. Washington DC: American Psychiatric Association Publishing; 2013.

2. Bisson JI, Cosgrove S, Lewis C, Roberts NP. Post-traumatic stress disorder. BMJ 2015;351:h6161.

3. Kessler RC, Sonnega A, Bromet E, Hughes M, Nelson CB. Posttraumatic stress disorder in the National Comorbidity Survey. Arch Gen Psychiatry 1995;52:1048-1060.

4. Atwoli L, Stein DJ, Koenen KC, McLaughlin KA. Epidemiology of posttraumatic stress disorder: prevalence, correlates and consequences. Curr Opin Psychiatr 2015;28:307-311.

5. Lim SW, Kim L. Sleep of PTSD. J Korean Neuropsychiatr Assoc 2005; 44:152-157.

6. Schlosberg A, Benjamin M. Sleep patterns in three acute combat fatigue cases. J Clin Psychiatry 1978;39:546-549.

7. Ross RJ, Ball WA, Sullivan KA, Caroff SN. Sleep disturbance as the hallmark of posttraumatic stress disorder. Am J Psychiatry 1989;146:696707.

8. Maher MJ, Rego SA, Asnis GM. Sleep disturbances in patients with post-traumatic stress disorder: epidemiology, impact and approaches to management. CNS Drugs 2006;20:567-591.

9. Spoormaker VI, Montgomery P. Disturbed sleep in post-traumatic stress disorder: secondary symptom or core feature? Sleep Med Rev 2008;12: 169-184.

10. Engdahl BE, Eberly RE, Hurwitz TD, Mahowald MW, Blake J. Sleep in a community sample of elderly war veterans with and without posttraumatic stress disorder. Biol Psychiatry 2000;47:520-525.

11. Germain A, Buysse DJ, Nofzinger E. Sleep-specific mechanisms underlying posttraumatic stress disorder: integrative review and neurobiological hypotheses. Sleep Med Rev 2008;12:185-195.

12. Krakow BJ, Ulibarri VA, Moore BA, McIver ND. Posttraumatic stress disorder and sleep-disordered breathing: a review of comorbidity research. Sleep Med Rev 2015;24:37-45.

13. Lavie P. Sleep disturbances in the wake of traumatic events. N Engl J Med 2001;345:1825-1832.

14. Raskind MA, Peskind ER, Hoff DJ, Hart KL, Holmes HA, Warren D, et al. A parallel group placebo controlled study of prazosin for trauma nightmares and sleep disturbance in combat veterans with post-traumatic stress disorder. Biol Psychiatry 2007;61:928-934.

15. Mysliwiec V, O’Reilly B, Polchinski J, Kwon HP, Germain A, Roth BJ.
Trauma associated sleep disorder: a proposed parasomnia encompassing disruptive nocturnal behaviors, nightmares, and REM without atonia in trauma survivors. J Clin Sleep Med 2014;10:1143-1148.

16. Mysliwiec V, Brock MS, Creamer JL, O’Reilly BM, Germain A, Roth BJ. Trauma associated sleep disorder: a parasomnia induced by trauma. Sleep Med Rev 2018;37:94-104.

17. Feemster JC, Smith KL, McCarter SJ, St Louis EK. Trauma-associated sleep disorder: a posttraumatic stress/REM sleep behavior disorder mash-up? J Clin Sleep Med 2019;15:345-349.

18. Elliott JE, Opel RA, Pleshakov D, Rachakonda T, Chau AQ, Weymann $\mathrm{KB}$, et al. Posttraumatic stress disorder increases the odds of REM sleep behavior disorder and other parasomnias in Veterans with and without comorbid traumatic brain injury. Sleep 2020;43:zsz237.

19. Kang SH, Yoon IY, Lee SD, Han JW, Kim TH, Kim KW. REM sleep behavior disorder in the Korean elderly population: prevalence and clinical characteristics. Sleep 2013;36:1147-1152.

20. Mahlknecht P, Seppi K, Frauscher B, Kiechl S, Willeit J, Stockner H, et al. Probable RBD and association with neurodegenerative disease markers: a population-based study. Mov Disord 2015;30:1417-1421.

21. Husain AM, Miller PP, Carwile ST. Rem sleep behavior disorder: potential relationship to post-traumatic stress disorder. J Clin Neurophysiol 2001;18:148-157.

22. Sohn SI, Kim DH, Lee MY, Cho YW. The reliability and validity of the Korean version of the Pittsburgh Sleep Quality Index. Sleep Breath 2012; 16:803-812.

23. Cho YW, Lee JH, Son HK, Lee SH, Shin C, Johns MW. The reliability and validity of the Korean version of the Epworth sleepiness scale. Sleep Breath 2011;15:377-384.

24. Hoddes E, Zarcone V, Smythe H, Phillips R, Dement WC. Quantification of sleepiness: a new approach. Psychophysiology 1973;10:431-436.

25. Cho YW, Song ML, Morin CM. Validation of a Korean version of the insomnia severity index. J Clin Neurol 2014;10:210-215.

26. Yu E, Ko Y, Sung G, Kwon J. Validation of the Korean version of dysfunctional beliefs and attitudes about sleep (K-DBAS-16). Kor J Clin Psychol 2009;28:309-320.

27. Sung G, Kim J, Park Y, Bai D, Lee S, Ahn H, et al. A study on the reliability and the validity of Korean version of the Beck Depression Inventory-II (BDI-II). J Korean Soc Biol Ther 2008;14:201-212.

28. Buysse DJ, Reynolds CF, Monk TH, Berman SR, Kupfer DJ. The Pittsburgh Sleep Quality Index: a new instrument for psychiatric practice and research. Psychiatry Res 1989;28:193-213.

29. Buysse DJ, Reynolds III CF, Monk TH, Hoch CC, Yeager AL, Kupfer DJ. Quantification of subjective sleep quality in healthy elderly men and women using the Pittsburgh Sleep Quality Index (PSQI). Sleep 1991;14: 331-338.

30. Berry RB, Wagner MH. Sleep Medicine Pearls, 3rd Edition. Philadelphia: Saunders, Elsevier; 2015.

31. Woodward SH, Bliwise DL, Friedman MJ, Gusman DF. Subjective versus objective sleep in Vietnam combat veterans hospitalized for PTSD. J Trauma Stress 1996;9:137-143.

32. Hurwitz TD, Mahowald MW, Kuskowski M, Engdahl BE. Polysomnographic sleep is not clinically impaired in Vietnam combat veterans with chronic posttraumatic stress disorder. Biol Psychiatry 1998;44:10661073.

33. Kobayashi I, Huntley E, Lavela J, Mellman TA. Subjectively and objectively measured sleep with and without posttraumatic stress disorder and trauma exposure. Sleep 2012;35:957-965.

34. Lee J, Chang M, Kim T. A survey study of psychological characteristics and post-traumatic stress symptoms in veterans who performed special mission : comparing anti-north secret agents, Vietnam veterans and normal control subjects. Rehabil Psychol 2016;23:1-20.

35. Magruder KM, Goldberg J, Forsberg CW, Friedman, MJ, Litz BT, Vaccarino V, et al. Long-term trajectories of PTSD in Vietnam-Era Veterans: the course and consequences of PTSD in twins. J Trauma Stress 2016;29:5-16. 
36. Huang J, Zhang J, Lam SP, Li SX, Ho CK, Lam V, et al. Amelioration of obstructive sleep apnea in REM sleep behavior disorder: implications for the neuromuscular control of OSA. Sleep 2011;34:909-915.

37. Kobayashi I, Boarts JM, Delahanty DL. Polysomnographically measured sleep abnormalities in PTSD: a meta-analytic review. Psychophysiology 2007;44:660-669.

38. Lavie P, Katz N, Pillar G, Zinger Y. Elevated awaking thresholds during sleep: characteristics of chronic war-related posttraumatic stress disorder patients. Biol Psychiatry 1998;44:1060-1065.

39. Breslau N, Roth T, Burduvali E, Kapke A, Schultz L, Roehrs T. Sleep in lifetime posttraumatic stress disorder: a community-based polysomnographic study. Arch Gen Psychiatry 2004;61:508-516.

40. Mellman TA, Bustamante V, Fins AI, Pigeon WR, Nolan B. REM sleep and the early development of posttraumatic stress disorder. Am J Psychiatry 2002;159:1696-1701.

41. Woodward S, Arsenault N, Michel G, Santerre C, Groves W, Stewart W. Polysomnographic characteristics of trauma-related nightmares. Sleep 2000;23:A356-A357.

42. Walker MP, van Der Helm E. Overnight therapy? The role of sleep in emotional brain processing. Psychol Bull 2009;135:731-748.

43. Garcia-Rill E. Disorders of the reticular activating system. Med Hypotheses 1997;49:379-387.

44. Jo S, Kim HW, Jeon JY, Lee SA. Protective effects of REM sleep without atonia against obstructive sleep apnea in patients with idiopathic REM sleep behavior disorder. Sleep Med 2019;54:116-120.

45. Yager TJ, Gerszberg N, Dohrenwend BP. Secondary traumatization in Vietnam Veterans' families. J Trauma Stress 2016;29:349-355.

46. Schenck CH, Boeve BF, Mahowfald MW. Delayed emergence of a parkinsonian disorder or dementia in $81 \%$ of older men initially diagnosed with idiopathic rapid eye movement sleep behavior disorder: a 16-year update on a previously reported series. Sleep Med 2013;14:744-748.

47. Benca RM, Okawa M, Uchiyama M, Ozaki S, Nakajima T, Shibui K, et al. Sleep and mood disorders. Sleep Med Rev 1997;1:45-56.

48. Winkelman JW, James L. Serotonergic antidepressants are associated with REM sleep without atonia. Sleep 2004;27:317-321.

49. Frauscher B, Jennum P, Ju YES, Postuma RB, Arnulf I, De Cock VC, et al. Comorbidity and medication in REM sleep behavior disorder: a multicenter case-control study. Neurology 2014;82:1076-1079. 